Article

\title{
Evaluation of Uncertainties in the Design Process of Complex Mechanical Systems
}

\author{
Francesco Villecco * and Arcangelo Pellegrino \\ Department of Industrial Engineering, University of Salerno, Via Giovanni Paolo II 132, 84084 Fisciano, Italy; \\ apellegrino@unisa.it \\ * Correspondence: fvillecco@unisa.it; Tel.: +39-089-964324
}

Received: 20 July 2017; Accepted: 31 August 2017; Published: 6 September 2017

\begin{abstract}
In this paper, the problem of the evaluation of the uncertainties that originate in the complex design process of a new system is analyzed, paying particular attention to multibody mechanical systems. To this end, the Wiener-Shannon's axioms are extended to non-probabilistic events and a theory of information for non-repetitive events is used as a measure of the reliability of data. The selection of the solutions consistent with the values of the design constraints is performed by analyzing the complexity of the relation matrix and using the idea of information in the metric space. Comparing the alternatives in terms of the amount of entropy resulting from the various distribution, this method is capable of finding the optimal solution that can be obtained with the available resources. In the paper, the algorithmic steps of the proposed method are discussed and an illustrative numerical example is provided.
\end{abstract}

Keywords: uncertainty; non-probabilistic entropy; fair division; design; complexity; multibody systems

\section{Introduction}

In industrial applications, the final design solution is almost always an engineering approximation, thus intrinsically prone to uncertainties. A viable approach for the analysis of the approximation in a design solution is based on the use of the non-probabilistic entropy. In the classic theories of Fisher and of Wiener-Shannon, the idea of information is a measure of probabilistic and repetitive events only. However, the concept is applicable beyond the probability field. In this respect, the Wiener-Shannon's axioms can be extended to non-probabilistic events so much so that it is possible to introduce a theory of information for non-repetitive events as a measure of the reliability of data. To this end, solutions consistent with the values of the design constraints may be selected by analyzing the complexity of the relation matrix and using the idea of information in the metric space. The final solution gives an entropic measure of epistemic uncertainties which can be then used in multibody system models analyzed by means of the axiomatic design. Multibody systems are the class of mechanical systems composed of rigid and/or flexible bodies interconnected by kinematic joints [1-10]. They are subjected to nonlinear external force fields and undergo large, finite translations and rotations [11-15]. Examples of multibody systems can be found in several engineering applications [16-20]. The complexity of the dynamic behavior of this family of constrained mechanical systems requires the development of advanced analysis and modeling tools to perform virtual prototyping in the multibody framework [21-27]. The problem is particularly challenging when control strategies for flexible multibody systems need to be designed [28-33]. In general, the design process gives the structure necessary to transform qualitative needs, often stated in non-engineering terms, into real products. In computer simulation of real problems, multiplicity of a data source may lead to a high level of error and, consequently, the final result can be considered only partially 
satisfactory. This process is carried out through the application of scientific knowledge to the problems. Based on a database of previous designs, the current design process generates several alternatives to be frequently evaluated. Usually, the design process is subdivided into phases with given specifications. Evaluations are required to determine whether the designer needs to go back to one or more phase. In the development of new industrial technology, the designer has the task of selecting among alternative solutions for complex systems. In this general process, besides a set of objective technical requirements, the designer must take into account some subjective factors that only he is able to quantify. The designer must also assess to what extent the selected alternatives fulfill the quantified targets. In particular, in the study of the dynamic behavior of multibody mechanical systems, it is necessary to develop a mathematical model with an appropriate objective function, which expresses the values of the combinations of possible outcomes as a single measure in terms of both cost and effectiveness. From a system design point of view, each subsystem is a system in its own right, i.e., it requires the fulfillment of a set of goals, organized following a hierarchic pattern, in which a single weight is assigned to each target according to the design relevance. In most common situations, a certain number of resources is not correctly allocated because the necessary values are not known or are estimated only in intervals. Consequently, imprecise data and imperfect knowledge may lead to uncertainties, and, therefore, there is the mathematical problem of the division of a quantity $\mathrm{Q}$ in a set of $\mathrm{n}$ fair values. If the data on distribution are not well-known, it is possible to use the principle of maximum entropy to achieve a consistent solution to the problem.

\section{Complexity}

In design research, the importance of explicitly considering uncertainty in the formulation of design is of fundamental importance for producing acceptable results. Multiple alternatives can yield uncertainties because of the lack of information. In this process, a good model helps to decide which alternative to take in order to achieve a final result that is acceptable. The best tools for obtaining good results are those that give a low quantity of uncertainties. In general, a set of interconnected components, interacting among each other in an organized way to achieve the same objective, constitute a system in a broad sense. Multibody mechanical systems represent a classical example of this general idea. Each system can be part of a super-system, i.e., a set of interacting systems. Therefore, to obtain a fast and good design necessitates analyzing the system complexity. Some projects may not need multiple sequences, while a large design may need to be analyzed in more layers. Each project may have its own organizational structure and subsystems. Complex systems are formed by subsystems which must operate in a coordinated way to achieve the desired goals. From a systems design point of view, each subsystem is a system in its own right and, therefore, each subsystem needs a set of requirements necessary to achieve the desired goals. Each requirement can be organized following a hierarchical scale, and its relevance is set by the designers assigning a weight in the hierarchy. The assignment of the weight to each element or system in the super-system is critical to establish a fair allocation of resources. An estimation of consistency of the individual resources must be conducted in order to give an overall assessment of the distribution.

\section{Uncertainty}

Uncertainty is a constant in engineering design. This is particularly true for multibody mechanical systems. Actually, conventional design methods suffer from the presence of uncertainty, so much so that new methods and new strategies have been recently introduced. By means of extensive theoretical research and virtual prototyping, a significant improvement can be obtained in the results within the framework of the new methods. The design process carried out under uncertainty is a difficult task because of both the lack of data and, more importantly, the lack of knowledge of the system under analysis. The effects of errors in input data and on the uncertainty of the model may be distinguished and quantified by the Bayesian inference. Choices made during the design with the Bayesian models are based on the theory that allows one to make optimal decisions under uncertainty. The goal of this design 
process is to maximize the expected utility of the project results, the being latter defined in terms related to the optimal experiment design, depending on the particular objective of the chosen product [34-36].

\section{Fair Distribution of Resources}

In most common situations, the belief is of no crisp because either its value is unknown or it is only known its range of existence [37]. In mathematical terms, the quantity $Q$ has to be divided in a set $\left\{q_{i}\right\}$ of an fair values with every element $q_{i} \geq 0$. If the distribution is fair, with $v_{i} \geq 0$, which is divided by the weight of the nth share, one can write:

$$
v_{1}+v_{2}+\ldots+v_{n}=1
$$

and $q_{i}=v_{i} Q$.

When the information on distribution is not completely well-known, it is possible to use the maximum entropy principle to obtain a solution of the problem. The model of the maximum entropy principle is inborn in Western philosophy and is the mathematical transcription of the ancient principle of wisdom: in the absence of information on events, the same belief holds for any of them. This statement is known as the Principle of Insufficient Reason of Laplace and Bernoulli: if there is no reason to believe that out of a set of possible, mutually exclusive events, no one event is more likely to occur than any other, then one should assume that all events are equally probable. This is a decision principle because, in the absence of a priori reason, one can assign an equal degree of belief to all the events. In presence of incomplete information, the maximum entropy principle assigns and completes the information. Using Shannon's definition, the entropy in a uniform (equally likely) distribution of probability assumes the maximum value. Therefore, one can write:

$$
\begin{gathered}
S(u)=-\sum_{1}^{n} p_{i} \ln p_{i} \\
u=\left(\frac{1}{n}, \frac{1}{n}, \ldots, \frac{1}{n}\right) \\
S(u)=\ln (n)
\end{gathered}
$$

On the basis of the Laplace-Bernoulli principle, one can assign degrees of belief to finite collections of events.

In a probability finite space $\Omega$ the measure of probability must observe Kolmogorov's axioms, which can be summarized as follows:

If in the probability space $(\Omega, A, P)$

$\Omega$ is a finite non-empty set;

$A$ is an $\sigma$-algebra off the subset in $\Omega$ of the events;

$P$ is a probability measure on $A$,

Then the applications of $P: A \rightarrow[0,1]$ must be under axioms:

$$
\begin{gathered}
P(\Omega)=1, P(\phi)=\phi, A_{n} \in A \forall n \\
A_{n} \cap A_{m}=\phi \\
m \neq n \Rightarrow P\left(\begin{array}{c}
\cup A_{n} \\
n
\end{array}\right)=\sum_{n} P\left(A_{n}\right)
\end{gathered}
$$

Now, if we analyze our problem in Kolmogorov's mathematical formalism [38], we can use his philosophy of probability and how he proposed to relate the mathematical formalism to the real world. With this formalism, we can use the tool of Shannon's entropy to measure the uncertainty of the division. 
When one knows only that the belief is included in intervals, an algorithm is required for the division of a quantity. Indicating with $n$ the number of intervals, a set of $\left\{v_{i}\right\}$ fair values under the condition $\sum_{i}^{n} v_{i}=1$ can be found. For the generic $v_{i} \geq 0$, one has $v_{i} \in\left[\underline{v_{i}}, \overline{v_{i}}\right]$, where $\underline{v_{i}}$ is a lower value and $\overline{v_{i}}$ is the upper value of the interval of $v_{i}$, and therefore:

$$
\sum_{1}^{n} \underline{v_{i}} \leq 1 \leq \sum_{1}^{n} \overline{v_{i}}
$$

The value $\underline{v_{i}}$ is the lower possible of its interval $d_{i}=\left|\underline{v_{i}}-\overline{v_{i}}\right|$. The value $\delta_{i}=\left|\sum_{1}^{n} \underline{v_{i}}-1\right|$ is the total value of uncertainty and must be distributed. The fair partition depends on the weight of intervals $d_{i}$. The weight $W$ of all intervals is $\sum_{1}^{n}\left|\underline{v_{i}}-\overline{v_{i}}\right|=W$. The values of $v_{i}$ are given by:

$$
v_{i}=\underline{v_{i}}+\left(1-\sum_{1}^{n} \underline{v_{i}}\right)\left(\overline{v_{i}}-\underline{v_{i}}\right) / \sum_{1}^{n}\left(\overline{v_{i}}-\underline{v_{i}}\right)
$$

In order to have consistent problems, this formula can be used for the distribution of a quantity included in intervals in an uncertain position. If one has a set of $n=(m+k+u)$ elements with $k$ values well-known, $m$ values included in intervals in uncertain position, and $u$ unknown values, in the distribution with $i \in[1, n]$ and

$$
n \in[1, \ldots, k, k+1, \ldots, m, m+1, \ldots, u, u+1, \ldots n]
$$

one cannot assign the Laplace-Bernoulli values because all of the information is not known. Only the values of $v_{k}$ are well-known. The values $(k+m)$ can be divided into two subsets, $S=\underline{S} \cup \bar{S}$, with the subset $\underline{S}$ indicating the lower values and the subset $\bar{S}$ denoting the upper values:

$$
\begin{aligned}
& \underline{S}=\left\{\underline{v}_{i}, \ldots, \underline{v}_{k}, \underline{v}_{k+1}, \ldots, \underline{v}_{m}\right\} \\
& \bar{S}=\left\{\bar{v}_{i}, \ldots, \bar{v}_{k}, \bar{v}_{k+1}, \ldots, \bar{v}_{m}\right\} \\
& \underline{\Delta}=1-\sum_{1}^{m} \underline{v}_{i} ; \bar{\Delta}=1-\sum_{1}^{m} \bar{v}_{i}
\end{aligned}
$$

a. One thus obtains the data, $\bar{\Delta}$ and $\underline{\Delta}$, for the distribution from the subset $\underline{S}$ and $\bar{S}$, while the sub-rules are:

b. If $\bar{\Delta}<0$ then $\underline{\mathrm{v}}=0, \mathrm{i} \in\{m+1, \ldots, u\}$.

If $\underline{\Delta}>0$ then the distribution is consistent.

c. If $\bar{\Delta}>0$ and $\underline{\Delta}>0$ then the value of the upper set $\underline{\Delta}$ determines the lower distributions and the one of the lower set $\underline{\Delta}$ determines the higher distribution (Table 1 ).

Table 1. Higher distribution.

\begin{tabular}{ccc}
\hline Well-Known Values & Values Included in Intervals & Values of the Laplace-Bernuille Distribution \\
\hline$p_{1}=v_{1}=\underline{v}_{1}=\bar{v}_{1}$ & $p_{k+1} \in\left[\underline{v}_{m}, \bar{v}_{m}\right]$ & $p_{m+1} \in[\bar{\Delta} / u, \underline{\Delta} / u]$ \\
$p_{k}=v_{k}=\underline{v}_{k}=\bar{v}_{k}$ & $p_{m} \in\left[\underline{v}_{m}, \bar{v}_{m}\right]$ & $p_{u} \in[\Delta / u, \underline{\Delta} / u]$ \\
\hline
\end{tabular}

Using the principle of maximum entropy, the fair distribution can be determined. In this paper, the use of the method by a very simple application reported in Table 2 is introduced.

The proposed method is new. It can be used in the distribution of a quantity in the presence of uncertainty in probabilistic and non-probabilistic problems. 
Table 2. Maximum entropy application.

\begin{tabular}{|c|c|c|c|c|c|c|c|c|c|}
\hline$v_{i}$ & $\underline{v}_{i}$ & $\bar{v}_{i}$ & $\left(\bar{v}_{i}-\underline{v}_{i}\right)$ & - & $v_{i}$ & $\underline{v}_{i}$ & $\bar{v}_{i}$ & $\left(\bar{v}_{i}-\underline{v}_{i}\right)$ & res \\
\hline 1 & 0.1 & 0.1 & 0 & crisp & 1 & 0.1 & 0.1 & 0 & 0.1 \\
\hline 2 & 0.1 & 0.3 & 0.2 & unce & 2 & 0.1 & 0.3 & 0.2 & 0.2 \\
\hline 3 & 0.2 & 0.4 & 0.2 & unce & 3 & 0.2 & 0.4 & 0.2 & 0.3 \\
\hline 4 & - & - & - & unkn & 4 & $\bar{\Delta} / 2=0.1$ & $\underline{\Delta} / 2=0.3$ & 0.2 & 0.2 \\
\hline 5 & - & - & - & unkn & 5 & $\bar{\Delta} / 2=0.1$ & $\underline{\Delta} / 2=0.3$ & 0.2 & 0.2 \\
\hline- & $\sum_{1}^{3} \underline{v}_{i}=0.4$ & $\sum_{1}^{3} \bar{v}_{i}=0.4$ & - & - & - & $\sum_{1}^{5} \underline{v}_{i}=0.6$ & $\sum_{1}^{5} \bar{v}_{i}=1.4$ & $\Sigma=0.8$ & $\Sigma=1$ \\
\hline- & $\bar{\Delta}=1$ & $\bar{v}_{i}=0.2$ & - & - & - & $1-\sum_{1}^{5}$ & $=0.4$ & - & - \\
\hline- & $\underline{\Delta}=1-$ & $i=0.6$ & - & - & - & $\sum_{1}^{5}\left(\bar{v}_{i}-\right.$ & )$=0.8$ & - & - \\
\hline
\end{tabular}

\section{Measure of the Information of a Distribution of Imprecise Data}

In the theories of Fisher and Wiener-Shannon, the idea of information is not only a measure only of probabilistic and repetitiveness events, but can also be used as a measure of the fairness of the complex distributions [39,40]. The use of the information axioms allows for the development of useful and applicable information models. For every event $t$ is possible to have a measure of information using the mathematical expression:

$$
J(A)=\frac{1}{d(A)}
$$

This definition of information has a natural application in the metric space. Norbert Wiener in Cybernetics gave the clearest definition of entropy:

$$
\text { Entropy }=-\log _{2} \frac{\text { measureo } f(a, b)}{\text { measureo } f(0,1)}
$$

The information can be evaluated by the probability and by the non-probabilistic measures of diameters. Thus, it is possible to have the measure of the information from non-probabilistic data. For the non-probabilistic information, instead of probability, it is possible to utilize a finite number of appropriate proportions, subject to a set of constraints that add up to 1 . Let $d_{1}, d_{2}, \ldots, d_{n}$ be non-negative real numbers, and let:

$$
\begin{cases}\sum_{i=1}^{n} d_{i} \neq 0 & \rho_{i}=\frac{d_{i}}{d_{1}+d_{2}+\ldots+d_{n}} \\ \sum_{i=1}^{n} \rho_{i}=1 & \rho_{i} \geq 0 \forall i\end{cases}
$$

One can use the following relation as a measure of information:

$$
J(\rho)=J\left(\rho_{1}, \rho_{2}, \ldots, \rho_{n}\right)=-\sum_{i=1}^{n} \rho_{i} \ln \rho_{i}
$$

Thus, $J(\rho)$ is maximum when $\rho_{1}=\rho_{2}=\ldots=\rho_{n}$, and $J(\rho)$ is minimum when, for every element $i$, only one number is different from zero. In the final result, there is the final distribution that also takes into account the uncertainty of data, with a fair distribution of imprecise data and a global measure of uncertainty. For example, one can write:

$$
h_{1}=\sum_{1}^{5}-\rho_{i} \ln \rho_{i}=-(0.1 \ln 0.1+0.2 \ln 0.2+0.3 \ln 0.3+0.2 \ln 0.2+0.2 \ln 0.2)=1.5 \text { bit }
$$

If the final distribution is uniform, then the result would be a maximum number of bits because the uniform distribution involves the maximum entropy value: 


$$
h_{\text {max }}=\sum_{1}^{5}-\rho_{i} \ln \rho_{i}=-\sum_{1}^{5} \frac{1}{5} \ln \frac{1}{5}=1.6 \mathrm{bit}
$$

Thus, it is possible to measure the entropy of the two distributions and to choose the best solution for our design.

\section{Conclusions}

The principal goal of the authors' research is the development of new methods for the design and the analysis of complex mechanical systems [41-51]. In general, the designer of complex systems is often called upon to select a suitable solution among several alternatives. For industrial engineering applications, this is particularly true in the design process of multibody mechanical systems composed of several bodies constrained by kinematic joints. In this challenging process, some subjective factors that are difficult to quantify analytically must be taken into account in order to judge which alternative will effectively fulfill the desired targets. When possible, in this complex process a mathematical model which includes an objective function can be developed in order to facilitate the design process using a single quantity expressing the performance of the combinations of all the possible outcomes in terms of cost and effectiveness. From a system design point of view, each subsystem is a system in its own right, i.e., it must achieve a set of goals organized in a hierarchical way by assigning a weight according to their relevance. The assignment of the weight for each element or each system in the super-system can be obtained using the fair distribution model. Using this methodology, the challenge is to establish the right distribution for a given problem. It is thus necessary to devise some mathematical tools to compare and evaluate two or more distributions. For this purpose, Wiener-Shannon's axioms can be extended to non-probabilistic events, so that it is possible to introduce a theory of information for non-repetitive events as a measure of the reliability of data. To this end, one can select solutions consistent with the values of the design constraints by analyzing the complexity of the relation matrix and using the idea of information in the metric space. Analyzing the alternatives by the amount of entropy resulting from the various distribution, this method allows one to find the optimal solution that can be obtained with the available resources. As shown in the paper, the principal algorithmic steps for a correct analysis are three, namely, the creation of the alternative solutions to meet the functional requirements of the project, the evaluation of alternatives based on the resource budget, and the entropic classification of the alternative solutions. In the paper, a simple numerical example is illustrated in order to demonstrate the effectiveness of the proposed methodology.

Author Contributions: The work was mainly developed by the first author (Francesco Villecco). The critical review conducted by the second author (Arcangelo Pellegrino) is greatly appreciated.

Conflicts of Interest: The authors declare no conflict of interest.

\section{References}

1. Batou, A.; Soize, C. Rigid multibody system dynamics with uncertain rigid bodies. Multibody Syst. Dyn. 2012, 27, 285-319. [CrossRef]

2. De Simone, M.C.; Guida, D. Dry Friction Influence on Structure Dynamics. In Proceedings of the 5th ECCOMAS Thematic Conference on Computational Methods in Structural Dynamics and Earthquake Engineering, Crete, Greece, 25-27 May 2015; pp. 4483-4491.

3. Guida, D.; Pappalardo, C.M. A New Control Algorithm for Active Suspension Systems Featuring Hysteresis. FME Trans. 2013, 41, 285-290.

4. Borelli, D.; Devia, F.; Lo Cascio, E.; Schenone, C.; Spoladore, A. Combined production and conversion of energy in an urban integrated system. Energies 2016, 9, 817. [CrossRef]

5. Pappalardo, C.M.; Wang, T.; Shabana, A.A. On the Formulation of the Planar ANCF Triangular Finite Elements. Nonlinear Dyn. 2017. [CrossRef]

6. Chung, W.-H.; Kremer, G.E.O.; Wysk, R.A. A dynamic programming method for product upgrade planning incorporating technology development and end-of-life decision. J. Ind. Prod. Eng. 2017, 34, 30-41. [CrossRef] 
7. Pappalardo, C.M.; Guida, D. Adjoint-Based Optimization Procedure for Active Vibration Control of Nonlinear Mechanical Systems. J. Dyn. Syst. Meas. Control 2017, 139, 081010. [CrossRef]

8. Kulkarni, S.; Pappalardo, C.M.; Shabana, A.A. Pantograph/Catenary Contact Formulations. J. Vib. Acoust. 2017, 139, 011010. [CrossRef]

9. Frimpong, S.; Thiruvengadam, M. Multibody Dynamic Stress Simulation of Rigid-Flexible Shovel Crawler Shoes. Minerals 2016, 6, 61. [CrossRef]

10. Pappalardo, C.M.; Wallin, M.; Shabana, A.A. A New ANCF/CRBF Fully Parametrized Plate Finite Element. J. Comput. Nonlinear Dyn. 2017, 12, 031008. [CrossRef]

11. Torres-Moreno, J.L.; Blanco-Claraco, J.L.; Giménez-Fernández, A.; Sanjurjo, E.; Naya, M.Á. Kinematic and Dynamic-State Estimation for Constrained Multibody Systems Based on IMUs. Sensors 2016, 16, 333. [CrossRef] [PubMed]

12. Pappalardo, C.M.; Guida, D. Control of Nonlinear Vibrations using the Adjoint Method. Meccanica 2016. [CrossRef]

13. Park, K.; Kremer, G.E.O. Assessment of static complexity in design and manufacturing of a product family and its impact on manufacturing performance. Int. J. Prod. Econ. 2015, 169, 215-232. [CrossRef]

14. Pappalardo, C.M.; Yu, Z.; Zhang, X.; Shabana, A.A. Rational ANCF Thin Plate Finite Element. J. Comput. Nonlinear Dyn. 2016, 11, 051009. [CrossRef]

15. Wagner, C.; Krinner, A.; Thümmel, T.; Rixen, D. Full Dynamic Ball Bearing Model with Elastic Outer Ring for High Speed Applications. Lubricants 2017, 5, 17. [CrossRef]

16. Pappalardo, C.M.; Patel, M.D.; Tinsley, B.; Shabana, A.A. Contact Force Control in Multibody Pantograph/Catenary Systems. Proc. Inst. Mech. Eng. Part K J. Multibody Dyn. 2016, 230, 307-328. [CrossRef]

17. Mathijssen, G.; Cherelle, P.; Lefeber, D.; Vanderborght, B. Concept of a Series-Parallel Elastic Actuator for a Powered Transtibial Prosthesis. Actuators 2013, 2, 59-73. [CrossRef]

18. Pappalardo, C.M. A Natural Absolute Coordinate Formulation for the Kinematic and Dynamic Analysis of Rigid Multibody Systems. Nonlinear Dyn. 2015, 81, 1841-1869. [CrossRef]

19. Guida, D.; Pappalardo, C.M. Control Design of an Active Suspension System for a Quarter-Car Model with Hysteresis. J. Vib. Eng. Technol. 2015, 3, 277-299.

20. Guo, Z.; Liu, H.; Zhang, D.; Yang, J. Green Supplier Evaluation and Selection in Apparel Manufacturing Using a Fuzzy Multi-Criteria Decision-Making Approach. Sustainability 2017, 9, 650.

21. Guida, D.; Pappalardo, C.M. Forward and Inverse Dynamics of Nonholonomic Mechanical Systems. Meccanica 2014, 49, 1547-1559. [CrossRef]

22. Li, M.; Yuan, M. An Approach to the Match between Experts and Users in a Fuzzy Linguistic Environment. Information 2016, 7, 22. [CrossRef]

23. Guida, D.; Nilvetti, F.; Pappalardo, C.M. Instability Induced by Dry Friction. Int. J. Mech. 2009, 3, 44-51.

24. Guida, D.; Nilvetti, F.; Pappalardo, C.M. Dry Friction Influence on Cart Pendulum Dynamics. Int. J. Mech. 2009, 3, 31-38.

25. Villecco, F.; Pellegrino, A. Entropic Measure of Epistemic Uncertainties in Multibody System Models by Axiomatic Design. Entropy 2017, 19, 291. [CrossRef]

26. Guida, D.; Nilvetti, F.; Pappalardo, C.M. Parameter Identification of a Two Degrees of Freedom Mechanical System. Int. J. Mech. 2009, 3, 23-30.

27. Guida, D.; Pappalardo, C.M. Sommerfeld and Mass Parameter Identification of Lubricated Journal Bearing. WSEAS Trans. Appl. Theor. Mech. 2009, 4, 205-214.

28. Pappalardo, C.M.; Patel, M.; Tinsley, B.; Shabana, A.A. Pantograph/Catenary Contact Force Control. In Proceedings of the ASME 2015 International Design Engineering Technical Conferences and Computers and Information in Engineering Conference, Boston, MA, USA, 2-5 August 2015; pp. 1-11.

29. Pappalardo, C.M.; Guida, D. On the use of Two-dimensional Euler Parameters for the Dynamic Simulation of Planar Rigid Multibody Systems. Arch. Appl. Mech. 2017. [CrossRef]

30. Yang, W.; Jiang, D.; Han, T. Effects of Tooth Breakage Size and Rotational Speed on the Vibration Response of a Planetary Gearbox. Appl. Sci. 2017, 7, 678. [CrossRef]

31. Pappalardo, C.M.; Wang, T.; Shabana, A.A. Development of ANCF Tetrahedral Finite Elements for the Nonlinear Dynamics of Flexible Structures. Nonlinear Dyn. 2017. [CrossRef] 
32. Concilio, A.; De Simone, M.C.; Rivera, Z.B.; Guida, D. A new semi-active suspension system for racing vehicles. FME Trans. 2017, 45, 578-584.

33. Quatrano, A.; De Simone, M.C.; Rivera, Z.B.; Guida, D. Development and implementation of a control system for a retrofitted CNC machine by using Arduino. FME Trans. 2017, 45, 565-571.

34. Suh, N.P. The Principles of Design; Oxford University Press: New York, NY, USA, 1990.

35. Wiener, N. Cybernetics. Sci. Am. 1948, 179, 14-18. [CrossRef] [PubMed]

36. Pahl, G.; Beitz, W.; Feldhusen, J.; Grote, K.H. Engineering Design; Springer: London, UK, 2007.

37. Yager, R.R.; Kreinovich, V. Fair Division under Interval Uncertainty. Int. J. Uncertain. Fuzz. 2000, 8, 611-618. [CrossRef]

38. Tikhomirov, V.M. Selected Works of A. N. Kolmogorov, 1st ed.; Springer: Dordrecht, The Netherlands, 1991.

39. Pappalardo, M. Information in Metric Space. J. Mater. Process. Tech. 2004, 157, 228-231. [CrossRef]

40. Donnarumma, A.; Pappalardo, M.; Pellegrino, A. Measure of independence in soft design. J. Mater. Process. Technol. 2002, 124, 32-35. [CrossRef]

41. Formato, A.; Ianniello, D.; Villecco, F.; Lenza, T.L.L.; Guida, D. Design Optimization of the Plough Working Surface by Computerized Mathematical Model. Emir. J. Food Agric. 2017, 29, 36-44. [CrossRef]

42. Sena, P.; d'Amore, M.; Pappalardo, M.; Pellegrino, A.; Fiorentino, A.; Villecco, F. Studying the Influence of Cognitive Load on Driver's Performances by a Fuzzy Analysis of Lane Keeping in a Drive Simulation. IFAC Proc. Vol. 2013, 46, 151-156. [CrossRef]

43. Sena, P.; Attianese, P.; Pappalardo, M.; Villecco, F. FIDELITY: Fuzzy Inferential Diagnostic Engine for on-LIne supporT to phYsicians. In Proceedings of the 4th International Conference on the Development of Biomedical Engineering, Ho Chi Minh City, Vietnam, 8-10 January 2012; IFMBE Proceedings. Springer: Berlin, Germany, 2013; pp. 396-400.

44. Sena, P.; Attianese, P.; Carbone, F.; Pellegrino, A.; Pinto, A.; Villecco, F. A Fuzzy Model to Interpret Data of Drive Performances from Patients with Sleep Deprivation. Comput. Math. Methods Med. 2012, 2012, 868410. [CrossRef] [PubMed]

45. Sansone, F.; Picerno, P.; Mencherini, T.; Villecco, F.; D’Ursi, A.M.; Aquino, R.P.; Lauro, M.R. Flavonoid Microparticles by Spray-drying: Influence of Enhancers of the Dissolution Rate on Properties and Stability. J. Food Eng. 2011, 103, 188-196. [CrossRef]

46. Pellegrino, A.; Villecco, F. Design Optimization of a Natural Gas Substation with Intensification of the Energy Cycle. Math. Probl. Eng. 2010, 2010, 294102. [CrossRef]

47. Zhai, Y.; Liu, L.; Lu, W.; Li, Y.; Yang, S.; Villecco, F. The Application of Disturbance Observer to Propulsion Control of Sub-mini Underwater Robot. In Proceedings of the ICCSA 2010 International Conference on Computational Science and Its Applications, Fukuoka, Japan, 23-26 March 2010; Lecture Notes in Computer Science. Springer: Berlin, Germany, 2010; pp. 590-598.

48. Ghomshei, M.; Villecco, F.; Porkhial, S.; Pappalardo, M. Complexity in Energy Policy: A Fuzzy Logic Methodology. In Proceedings of the 6th International Conference on Fuzzy Systems and Knowledge Discovery, Tianjin, China, 14-16 August 2009; IEEE: Los Alamitos, CA, USA; Volume 7, pp. 128-131.

49. Ghomshei, M.; Villecco, F. Energy Metrics and Sustainability. In Proceedings of the International Conference on Computational Science and Its Applications, Seoul, Korea, 29 June-2 July 2009; Lecture Notes in Computer Science. Springer: Berlin, Germany, 2009; pp. 693-698.

50. Zhang, Y.; Li, Z.; Gao, J.; Hong, J.; Villecco, F.; Li, Y. A method for designing assembly tolerance networks of mechanical assemblies. Math. Probl. Eng. 2012, 2012, 513958. [CrossRef]

51. Gao, Y.; Villecco, F.; Li, M.; Song, W. Multi-Scale Permutation Entropy Based on Improved LMD and HMM for Rolling Bearing Diagnosis. Entropy 2017, 19, 176. [CrossRef]

(C) 2017 by the authors. Licensee MDPI, Basel, Switzerland. This article is an open access article distributed under the terms and conditions of the Creative Commons Attribution (CC BY) license (http:/ / creativecommons.org/licenses/by/4.0/). 